\title{
THOUGHTS ON THE FUTURE OF PHOTOGRAPHIC CATALOGUES
}

\author{
HARLEY WOOD
}

Sydney Observatory, New South Wales, Australia

\begin{abstract}
The current astrometric programmes of zone photography and minor planet observations at the Sydney Observatory are reviewed. Future instrumental requirements and resources needed for zone catalogue work are discussed.
\end{abstract}

At Sydney Observatory zone photography of the southern sky began with the object of repeating, with better means, our astrographic zone $-51^{\circ}$ to $-65^{\circ}$ and possibly the zone $-64^{\circ}$ to the South Pole, originally allotted to Melbourne Observatory but largely completed and published from Sydney. The intention has been enlarged to embrace the whole southern sky. The camera is based on a Taylor, Taylor and Hobson lens of focal length $1776.6 \mathrm{~mm}$ (scale $116^{\prime \prime} \mathrm{mm}^{-1}$ ) and there is no deterioration of image quality over a field $6^{\circ} \times 6^{\circ}$. On a plate $20 \mathrm{~cm} \times 20 \mathrm{~cm}$ the measurable area is over $5^{\circ} \times 5^{\circ}$ so that taking zone centres 2.5 apart in declination and with right ascensions appropriately spaced there is full overlap in both coordinates. All exposures are made through a coarse diffraction grating.

So far all of the zones centred at declinations from -43.5 to -63.5 have been completely photographed and $-41^{\circ}$ and $-66^{\circ}$ are virtually complete, and in fact would have been so except for some rejections. Zones at -38.5 and -68.5 have been commenced and will go ahead immediately after the IAU Assembly.

Results of observations of selected minor planets give data relevant to catalogues of star places. At Sydney we have made observations over long arcs of such minor planets since 1955 and I quote from some results compiled by W. H. Robertson. Each plate taken has four exposures separated in declination. The results are always subjected to comparison of two kinds. First the means for all four images are taken with two separate groups of reference stars and second the mean result, using all reference stars simultaneously, from the first two exposures is compared with the result from the last two exposures. Each comparison implies a standard error for the result from the whole plate (all images and all stars). The first way has continuously through the whole period given standard deviation \pm 0 ".23 and the second way \pm 0 " 09 . Of course the second method eliminates completely the effect of errors in the star places and largely the effects of emulsion shift, metric properties of the image field and of errors in the measuring machine but not others, chiefly pointing errors. We have always tended to regard the difference between the two results as due to defects in the proper motions used to bring the star places to the epoch of the plate.

In 1955 and 1956, initiating during a visit by Dirk Brouwer, the plates on which a series of Yale Catalogues (e.g. Hoffleit, 1967) are based were taken at Sydney Observatory. The publication of these catalogues enables the plates in the appropriate area 
to be reduced again with contemporary star places. This does not affect the second class of comparison but the comparison using two sets of reference stars separately now implies a standard deviation \pm 0 ".09.

Recently we received from V. Orelskaya of the Institute of Theoretical Astronomy ephemerides of selected minor planets depending on observations collected from many sources. The deviations $(O-C)$ of our results from these ephemerides make possible another estimate of errors. The deviations usually keep nearly the same value over a long arc due possibly to a system in the catalogue from which the reference stars came or to a need for improvement of the orbit. A correction for this systematic difference having been applied the deviations from the ephemeris imply a standard error in each coordinate \pm 0 ".23 for the original results and \pm 0 ".07 for results using the contemporary star places.

The defects in the proper motions are verified and in fact a new reduction using the older catalogues but neglecting proper motion entirely leads in about $40 \%$ of cases to better agreement between results on one plate from the two sets of reference stars. These observations are made in areas of the sky not usually regarded as having the worst places and the need for continued positional work of quality is underlined.

It is scarcely necessary to enlarge on, or even to mention, to a group of astrometrists the fundamental part played by positional astronomy in many general activities of astronomy and astrophysics. It provides for the identification and recognition of many objects, for the observations needed in celestial mechanics including space science. Proper motions, obtained by positional techniques, provide a sieve to identify objects of various classes, for example those belonging to a cluster or association or those near to us in space, and form a part of the basic data needed in galactic kinematics. However there is plenty of evidence that it is necessary that it should be said with emphasis by the positional astronomers. At present there are at least three large telescopes, each costing perhaps 15 million dollars, under construction for use in the Southern Hemisphere. Much more is of course offered to space science. Half the cost of one such telescope devoted to positional astronomy would make a very great contribution to astronomy as a whole.

The same care and effort in design and choice of site (and comparable resources for construction) devoted to a great telescope needs to be available for positional projects. Due to the devotion of some positional astronomers progress on the design side is quite appreciable as revealed in reports of development of the Automatic Transit Circle at Washington and in the draft reports prepared by Fricke and Vasilevskis as presidents of IAU Commissions 8 and 24. This would doubtless be greater if there were assured prospects for construction and siting for most efficient use of the additional resources. The question as to the direction in which progress should be sought needs to be raised.

Leaving aside the means of solution (see for example Fricke, 1972) the problems of optical astrometry in fact remain much the same; to increase the accuracy of positions and proper motions and, within the best standard of accuracy, to establish a good self-consistent fundamental system over the whole sky and to relate through 
good proper motions observations both past and present to the same system which can be carried into the future. In some areas the relation between the fundamental system and the photographic zone catalogues (Fricke, 1972) remains weak. It seems important that the problem should be considered on a whole sky, all magnitude basis.

The availability of overlapping techniques which require fewer reference stars (for example Eichhorn and Gatewood, 1967) for reduction of measurements for photographic catalogues can work in two directions. They should mean on the one hand that effort devoted to meridian programmes might be directed less to providing a mass of observations of many stars and more to concentrating on the fundamental character of the observations and on the other hand that smaller fields, and longer focal lengths, can be used in the photography.

Longer focal lengths for this kind of work, (although suggested more than once), have not been much exploited, except in restricted areas (Eichhorn et al., 1970) where the success has been conspicuous. Their use would lead to greater accuracy through reduction of the influence of particularly measuring errors and emulsion shift. To estimate this in the conditions in which catalogues work is done, with measurements made over the whole plate, the model for the emulsion shifts needs to be carefully considered and the experience of Eichhorn and his collaborators who obtained a relative accuracy of 'the order of 0 ". 01 ', supports the use of larger focal lengths. Part of the accuracy was, of course, due to redundancy of material and advanced methods of reduction but it could not have been produced by the use of plate material at a scale of about $100^{\prime \prime} \mathrm{mm}^{-1}$ such as is commonly used for catalogue work.

Then what sort of instrument should be specified? Suppose that the largest plate size that can be tolerated is $40 \mathrm{~cm} \times 40 \mathrm{~cm}$ and the smallest field $4^{\circ} \times 4^{\circ}$. Allowing for an unmeasured border around the plate edge this leads to a focal length of over $500 \mathrm{~cm}$ and a scale $40^{\prime \prime} \mathrm{mm}^{-1}$, greater by a factor of 2.5 over frequently used scales.

Then the optical system needs to be considered. Although there are differing views on this, it does seem that a refracting system is to be desired. Luyten who has had such admirable success in the use of a Schmidt camera for measurement of proper motion nevertheless says (Luyten and La Bonte, 1972) that 'absolute' positions are "certainly not as good as can be obtained from Carte-du-Ciel plates (even allowing for the slight difference of scale)" and Dieckvoss (1972) speaking of the solution for positions on whole Schmidt plates says that if an observer "has to use Schmidt plates for photographic astrometry he has to restrict himself to rather small areas". In cases where experiments have been made with Schmidt cameras one usually finds that the results are not competitive or the area used has been restricted. There can be no doubt of the very real application of the Schmidt camera in the latter case.

However it is most important that there should be no compromise with the quality of the optical system. The images must be good over the whole field which must have excellent metric properties. The difficulty of arranging this depends on the magnitude limit sought but an aperture of less than $40 \mathrm{~cm}$ should be adequate. With the consequent aperture ratio of about $f / 13$ the lens designer should have a quite tractable problem. Estimates have been made of the astrometric accuracy as a function of scale but 
all that it seems necessary to say is that the standard error should for the first time come down to about 0 ".05.

In the past the measurement has been the barrier to quick progress in forming photographic catalogues. This need no longer be the case since measuring rates of 500 stars per hour (Reddish, 1972) are reported and even higher (Kibblewhite, 1972) projected. With a new machine for astrometry to a magnitude limit of say 12 and able to measure an area $40 \mathrm{~cm}$ it ought to be quite possible to keep up with the output of two cameras, one in each hemisphere.

The design of the telescope mounting and accessories too needs to provide for efficient working. It should be quite possible to arrange for all settings for each plate centre to be available in machine readable form and made by the turn of a switch and for the guide star to be found and followed automatically. The observer would then merely need to load and unload the plate holders. With two such a telescopes set up at well selected sites it would be possible to cover the sky in a period short compared with what we have been used to in the past.

\section{References}

Dieckvoss, W.: 1972, Conf. on the Role of Schmidt Telescopes in Astronomy, Hamburger Sternwarte, p. 43. Eichhorn, H. and Gatewood, G. D.: 1967, Astron. J. 72, 1191.

Eichhorn, H., Googe, W. D., Lukac, C. F., and Murphy, J. K.: 1970, Mem. Roy. Astron. Soc. 73, 125. Fricke, W.: 1972, Ann. Rev. Astron. Astrophys. 10, 101.

Hoffleit, D.: 1967, Trans. Astron. Obs. Yale Univ. 28.

Kibblewhite, E. J.: 1972, Observatory 92, 221.

Luyten, W. J. and La Bonte, A. E.: 1972, Conf. on the Role of Schmidt Telescopes in Astronomy, Hamburger Sternwarte, p. 35.

Reddish, V. C.: 1972, Observatory 92, 220.

\section{DISCUSSION}

Strand: I wonder if you have overlooked the high cost of building a machine measuring $40 \mathrm{~cm} \times 40 \mathrm{~cm}$. I estimate it to cost between $\$ 500 \mathrm{~K}$ and $\$ 750 \mathrm{~K}$, if not more.

Wood: No. This could well be among the items provided from the resources taken to be equivalent to half the cost of a large reflector.

Not all of these resources are allotted in the paper. What about adding two meridian circles to the two cameras and one measuring machine mentioned? This would leave enough for site selection and development and if there were a surplus it could be spent on an astrometric reflector. I find it hard to imagine expenditure on astronomy which would give better value per dollar.

Eichhorn: I agree that doing zone catalogue work with a telescope that gives a scale of $40^{\prime \prime} \mathrm{mm}^{-1}$ would give a much better return in accuracy per effort than the present $100^{\prime \prime} \mathrm{mm}^{-1}$ which was first used by Schlesinger and has since become almost a 'magic number'. Increasing the focal length is the easiest way to achieve higher positional accuracy, and it is remarkable that this has not been used more frequently.

Vasilevskis: Would it not be better to use the astrographs already at Lick and El Leoncito for cataloguing work? The scale is favourable at $55^{\prime \prime} \mathrm{mm}^{-1}$.

Wood: Well, I did think of this but, by a small margin, decided not to mention it. Perhaps I was wrong. If the telescopes were available for the necessary series of plates, close consideration would need to be given to the question.

Eichhorn: In answer to Vasilevskis' remark, $55^{2}=3025$, and $40^{2}=1600$, so that the weights of a position obtained by telescopes with scales of $40^{\prime \prime} \mathrm{mm}^{-1}$ and $55^{\prime \prime} \mathrm{mm}^{-1}$ would be approximately as $2: 1$. 